\title{
Editorial: Advances in Autoimmune Myasthenia Gravis
}

\author{
Anna Rostedt Punga ${ }^{1}$, Linda Kusner ${ }^{2}$, Sonia Berrih-Aknin ${ }^{3}$ and Rozen Le Panse ${ }^{3 *}$ \\ 'Department of Neuroscience, Clinical Neurophysiology, Uppsala University, Uppsala, Sweden, ${ }^{2}$ Department of \\ Pharmacology and Physiology, The George Washington University, Washington, DC, United States, ${ }^{3}$ Sorbonne University, \\ INSERM, Association Institute of Myology, Center of Research in Myology, Paris, France
}

Keywords: autoantibodies, CD4 T cells, thymus, therapy, miRNA

\section{Editorial on the Research Topic}

\section{Advances in Autoimmune Myasthenia Gravis}

Myasthenia gravis (MG) is an autoimmune neuromuscular disorder characterized by impaired neuromuscular transmission, which causes fluctuating fatigable muscle weakness. MG is a prototypical autoimmune disease with well-defined autoantibodies that target the neuromuscular junction. The majority of MG patients have autoantibodies against the acetylcholine receptor $(\mathrm{AChR})$ and a smaller proportion of patients have autoantibodies against the muscle-specific tyrosine kinase (MuSK) or the low-density lipoprotein-related protein 4 (LRP4) (Borges and Richman) (1). Furthermore, several other antigenic targets, such as agrin, Kv1.4 potassium channel, rapsyn, cortactin, acetylcholinesterase (AChE), collagen Q (ColQ), and collagen XIII, have been

OPEN ACCESS

Edited and reviewed by: Betty Diamond,

Feinstein Institute for Medical

Research, United States

*Correspondence:

Rozen Le Panse

rozen.lepanse@upmc.fr

Specialty section:

This article was submitted to Autoimmune and Autoinflammatory Disorders,

a section of the journal

Frontiers in Immunology

Received: 18 June 2020

Accepted: 24 June 2020

Published: 28 August 2020

Citation:

Punga AR, Kusner L, Berrih-Aknin S and Le Panse R (2020) Editorial:

Advances in Autoimmune Myasthenia Gravis. Front. Immunol. 11:1688. doi: 10.3389/fimmu.2020.01688 reported. Although the pathogenicity and specificity of these autoantibodies for MG have not been fully characterized, their presence could help in better understanding the variability in disease severity. Moreover, they could provide a diagnostic/prognostic value for the management of MG patients (Fichtner et al.; Lazaridis and Tzartos).

The existence of B cells, which produce autoantibodies, is dependent on the interaction with $\mathrm{CD}^{+}{ }^{+} \mathrm{T}$ cells-both are key factors in MG. Thymic regulatory $\mathrm{T}$ cells are not efficiently suppressive, and $\mathrm{T}$ helper cells are resistant to suppression (2). Phenotypic variation of regulatory $\mathrm{T}$ cells and functional impairment are more pronounced in the thymus than in peripheral cells (Truffault et al.). Thymic epithelial cells from MG patients appear to play a central role in $\mathrm{CD}^{+}{ }^{+} \mathrm{T}$ cell defect via the release of soluble factors, such as TSLP (Thymic Stromal LymphoPoietin) (Truffault et al.). T helper (Th) 1, Th2, Th17, and T follicular helper (Tfh) cells are involved in MG pathogenic mechanisms. An increase in the levels of interleukin (IL)-21, IL-4, IL-10, and IL-17A is observed in $\mathrm{CD}^{+}{ }^{+} \mathrm{T}$ peripheral cells in $\mathrm{AChR}$ antibody seropositive $\left(\mathrm{AChR}^{+}\right) \mathrm{MG}$, as compared to healthy controls (Çebi et al.). Among CD4 ${ }^{+} \mathrm{T}$ cells, the percentage of Th17 cells is increased in $\mathrm{AChR}+$ MG patients. ICOS (Inducible T-cell COStimulator) and PD-1 (Programmed cell death protein 1), two molecules associated with Tfh cell function, are also highly expressed on $\mathrm{CD}^{+}{ }^{+} \mathrm{CXCR} 5^{+}$ Tfh cells in AChR+ MG. Tfh cells can be stratified in Tfh regulatory cells, or Tfh1, Tfh2, and Tfh 17 cells that differentially affect B-cell differentiation. In AChR+ MG patients, the percentage of peripheral Tfh17 cells (CXCR3 ${ }^{-} \mathrm{CCR}^{+} \mathrm{CD} 4^{+} \mathrm{T}$ cells) is also increased but Tfh1 and Tfh2 cells remain unaffected. Some of these changes are also observed to a lesser degree in AChR- MG patients (Çebi et al.) and in MuSK antibody seropositive $\left(\mathrm{MuSK}^{+}\right.$) MG patients (3). Immunosuppressive treatments commonly used in MG do not affect these cells but enhance IL-10 in CD4 T cells (Çebi et al.), and also B cells (4), suggesting a role of IL-10 in favoring immuno-regulatory mechanisms. 
Classical treatments of MG include chronic treatments such as AChE inhibitors and general immunosuppressive drugs (5), thymectomy (6), as well as acute treatment for deterioration such as intravenous immunoglobulin and plasmapheresis (5). A new line of molecules is now available and is used for refractory MG. One of these is Rituximab, an anti-CD20 Bcell depleting monoclonal antibody which is often used as a second-line of treatment in combination with conventional immunosuppressants. Rituximab seems more efficient in $\mathrm{MuSK}^{+}$ MG patients, with the reduction in MuSK antibodies being associated with clinical improvement (Marino et al.). Other therapies aiming at targeting B cells are also emerging (Huda).

Most recently, medications inhibiting the cleavage of the complement protein C5 have been evaluated in clinical trials. Eculizumab (a monoclonal antibody) has obtained authorization from the US Food and Drug Administration to be used in MG. A second generation of C5 inhibitor, Zilucoplan (a macrocyclic), has also recently entered clinical trials (Albazli et al.). Molecules blocking the function of FcRn are of interest for autoimmune diseases. Inhibition of FcRn reduces the ability to recycle IgGs and thereby removes them from circulation. Agents such as Efgartigimod (IgG1 Fc fragment) or Nicocalimab, Rozanolixizumab, and RCT-140 (monoclonal antibodies) are in clinical trials for MG (Gable and Guptill).

Other therapeutic approaches are still at the preclinical phase and have demonstrated beneficial effects on experimental MG (EAMG) rodent models. If oral or nasal administration of AChR fragments suppress autoimmunity in EAMG (Yamada et al.) (7), the recombinant extracellular domain of MuSK may also be effective in inducing oral tolerance in $\mathrm{MuSK}^{+}$ EAMG (Reuveni et al.). Oral tolerance is a phenomenon based on suppressing immune responses in the gut where microbiota could play a role. In that way, probiotics could balance the gut microbiota and have beneficial effects in EAMG (Rinaldi et al.).

Even though MG has been studied for a number of years, the understanding of the etiological mechanisms is still evolving. In $\mathrm{AChR}^{+} \mathrm{MG}$, the thymus is known to play a central role in disease onset either in the earlyonset form of the disease or in MG-associated thymoma (8). The analyses of enriched pathways from "omics" data might reveal new unexplored pathways central in MG development (Cron et al.; Yamada et al.). It is well-known that genetic predispositions exist in MG patients (9), however, additional epigenetic changes occur. The expression of small noncoding RNA, microRNA (miRNA), is dysregulated in the thymus of $\mathrm{AChR}^{+} \mathrm{MG}$ patients $(10,11)$ and could be involved in thymic changes associated with $\mathrm{MG}$, linked to thymic inflammation and ectopic germinal center development (Bortone et al.; Cron et al.). Circulating miRNAs are also potential biomarkers since they are differentially expressed in the serum of MG patients (Sabre et al.). Specific circulating miRNAs have been associated with $\mathrm{AChR}^{+}$and $\mathrm{MuSK}^{+} \mathrm{MG}$ subtypes and their expression is regulated by treatment with immunosuppression and thymectomy (Fiorillo et al.; Sabre et al.). Investigations into the triggering events that lead to MG are still needed. Sexual hormones can affect, for example miRNAs, and in addition, favor autoimmunity in women (Fiorillo et al.) (12). Environmental factors are also candidates for driving/perpetuating autoimmunity, such as pathogen infection, endocrinal disruptors, and microbiota changes.

Although MG is a relatively well-characterized autoimmune disease, recent studies shed light on the mechanisms of development of this pathology and, most importantly, make it possible to propose more effective tools for monitoring and more effective treatments with fewer side effects. This Research Topic, dedicated to autoimmune MG, addresses these different aspects, both with Original Research articles and Reviews of the literature.

\section{AUTHOR CONTRIBUTIONS}

AP, LK, SB-A, and RL wrote the editorial. All authors contributed to the article and approved the submitted version.

\section{REFERENCES}

1. Mantegazza R, Bernasconi P, Cavalcante P. Myasthenia gravis: from autoantibodies to therapy. Curr Opin Neurol. (2018) 31:517-25. doi: 10.1097/WCO.0000000000000596

2. Gradolatto A, Nazzal D, Truffault F, Bismuth J, Fadel E, Foti M, et al. Both Treg cells and Tconv cells are defective in the Myasthenia gravis thymus: roles of IL17 and TNF-alpha. J Autoimmun. (2014) 52:53-63. doi: 10.1016/j.jaut.2013. 12.015

3. Li Y, Guptill JT, Russo MA, Howard JF, Massey JM, Juel VC, et al. Imbalance in $\mathrm{T}$ follicular helper cells producing IL-17 promotes pro- in fl ammatory responses in MuSK antibody positive myasthenia gravis. J Neuroimmunol. (2020) 345:577279. doi: 10.1016/j.jneuroim.2020.5 77279

4. Yilmaz V, Maillard S, Truffault F, Bolgert F, Behin A, Regnard JF, et al. Regulatory B cells in myasthenia gravis are differentially affected by therapies. Ann Clin Transl Neurol. (2018) 5:1408-14. doi: 10.1002/ac n3.645
5. Wang S, Breskovska I, Gandhy S, Punga AR, Guptill JT, Kaminski HJ. Advances in autoimmune myasthenia gravis management. Expert Rev Neurother. (2018) 18:573-588. doi: 10.1080/14737175.2018.1491310

6. Wolfe GI, Kaminski HJ, Aban IB, Minisman G, Kuo HC, Marx A, et al. Long-term effect of thymectomy plus prednisone versus prednisone alone in patients with non-thymomatous myasthenia gravis: 2-year extension of the MGTX randomised trial. Lancet Neurol. (2019). 18:P25968. doi: 10.1016/S1474-4422(18)30392-2

7. Weiner HL. Induction of oral tolerance to the acetylcholine receptor for treatment of myasthenia gravis. J Clin Invest. (1999) 104:16678. doi: 10.1172/JCI8775

8. Marx A, Pfister F, Schalke B, Saruhan-Direskeneli G, Melms A, Ströbel P. The different roles of the thymus in the pathogenesis of the various myasthenia gravis subtypes. Autoimmun Rev. (2013) 12:87584. doi: 10.1016/j.autrev.2013.03.007

9. Giraud M, Vandiedonck C, Garchon HJ. Genetic factors in autoimmune myasthenia gravis. Ann N Y Acad Sci. (2008). 1132:180-92. doi: 10.1196/annals.1405.027 
10. Cron MA, Maillard S, Villegas J, Truffault F, Sudres M, Dragin N, et al. Thymus involvement in early-onset myasthenia gravis. Ann N Y Acad Sci. (2018) 1412:137-45. doi: 10.1111/nyas.13519

11. Sengupta M, Wang BD, Lee NH, Marx A, Kusner LL, Kaminski HJ. MicroRNA and mRNA expression associated with ectopic germinal centers in thymus of myasthenia gravis. PLoS ONE. (2018) 13:e0205464. doi: 10.1371/journal.pone.0205464

12. Dragin N, Bismuth J, Cizeron-Clairac G, Biferi MG, Berthault C, Serraf A, et al. Estrogen-mediated downregulation of AIRE influences sexual dimorphism in autoimmune diseases. J Clin Invest. (2016) 126:152537. doi: 10.1172/JCI81894
Conflict of Interest: The authors declare that the research was conducted in the absence of any commercial or financial relationships that could be construed as a potential conflict of interest.

Copyright $\odot 2020$ Punga, Kusner, Berrih-Aknin and Le Panse. This is an open-access article distributed under the terms of the Creative Commons Attribution License (CC $B Y)$. The use, distribution or reproduction in other forums is permitted, provided the original author(s) and the copyright owner(s) are credited and that the original publication in this journal is cited, in accordance with accepted academic practice. No use, distribution or reproduction is permitted which does not comply with these terms. 